\title{
Sexual function in uncircumcised women with fibromyalgia: A controlled, cross-sectional, single-center study
}

\author{
Original \\ Article \\ Ahmed Ragab Ahmed', Hossam Marouf Fathy ${ }^{3}$, Sara Abdel-Elgawad ${ }^{4}$, Ashraf Samir \\ Fahim $^{2}$
}

Departments of ${ }^{1}$ Andrology and STDs, ${ }^{2}$ Gynecology and Obstetrics, ${ }^{3}$ Rheumatology and Rehabilitation, Faculty of Medicine, Beni-Suef University, Beni-Suef, ${ }^{4}$ Ministry of Health and Population, Cairo, Egypt

\begin{abstract}
Background: Fibromyalgia (FM) is a non-articulate rheumatic syndrome characterized by chronic musculoskeletal pain. Objective: The aim was to determine the rate of sexual dysfunction in uncircumcised women with FM and to appraise factors associated with sexual dysfunction in affected women.

Patients and Methods: A total of 50 drug-naive uncircumcised women with FM and 50 pain-free matched controls were compared regarding the scores of Arabic female sexual function index and hospital anxiety and depression subscales (HADS-A and HADS-D). The affected women were evaluated for tender point count (TPC), pain level on a visual analog scale (VAS), and FM related difficulties via Fibromyalgia Impact Questionnaire (FIQ). Moreover, the partners of patients and controls were evaluated by the 5-item international index of erectile function (IIEF-5).

Results: The mean age of patients was $30.42 \pm 4.99$ years and controls was $31.4 \pm 5.35$ years, which was insignificantly different $(P=0.345)$. Overall, $68 \%$ of patients and $16 \%$ controls displayed abnormal FSFI total scores $(P<0.001)$. FSFI overall and domain scores were significantly lower among patients than controls $(P<0.001)$. HADS-A and HADS-D scores were significantly higher among patients $(11.6 \pm 4.68$ and $11.46 \pm 4.96$, respectively) than controls $(5 \pm 2.64$ and $2.26 \pm 1.68$, respectively; $P<0.001)$. IIEF-5 scores were significantly higher among partners of controls $(22.48 \pm 1.42)$ compared with those of patients $(19.78 \pm 2.33, P<0.001)$. FSFI showed significant $(P<0.001)$ negative correlations with HADS-A, HADS-D, FIQ, VAS, TPC, and duration of disease and significant $(P<0.001)$ positive correlations with frequency of intercourse and IIEF-5. Regression analysis revealed that anxiety, pain severity, and frequency of intercourse were the independent factors associated with sexual impairment in FM (95\% confidence interval lower -1.117, -2.374, and 1.185 and upper -0.455, -1.040, and 4.366, and $P<0.001,<0.001$, and 0.001 , respectively).

Conclusion: FM could disturb all dimensions of female sexuality. The relatively high frequency of sexual impairment reported here $(68 \%)$ was associated with anxiety, pain severity, and frequency of intercourse.
\end{abstract}

Key Words: Drug-naïve uncircumcised women, female sexual function index, fibromyalgia impact questionnaire, fibromyalgia, hospital anxiety and depression scale.

Received: 04 July 2020, Accepted: 21 August 2020.

Corresponding Author: Ahmed Ragab Ahmed, Department of Andrology and STDs, Faculty of Medicine, Beni-Suef University, Beni-Suef, Egypt, Tel.: +20 1009800611, E-mail: drahmedragab1981@gmail.com.

ISSN: 2090-6048, 2020

\section{INTRODUCTION}

Remarkably, the nature of the female sexual response is highly sophisticated, and many factors affect it, including psychosocial and physiologic factors ${ }^{[1]}$. Sexual function (SF) is an essential component of life. On the contrary, sexual dysfunction (SD) can have a negative effect on the well-being of an individual ${ }^{[2]}$. SD is characterized by a disturbance in the processes that characterize the sexual response cycle or by pain associated with sexual intercourse $\mathrm{e}^{[3]}$.

Fibromyalgia syndrome (FMS) is one of the most common chronic musculoskeletal pain disorders ${ }^{[4]}$.
The prevalence of FMS is thought to be between 2 and $4 \%{ }^{[5-8]}$, with the female-to-male ratio of $6-9 / 1^{[9,10]}$. FMS is defined by the American College of Rheumatology (ACR) as chronic widespread musculoskeletal pain $(\geq 3$ months) and excess tenderness on palpation of at least 11 of 18 tender point sites throughout the body (multiple tender points) ${ }^{[11]}$. The 2010 ACR diagnostic criteria for FMS encompassed two scales: the Widespread Pain Index (WPI) and the Symptom Severity (SS) scale. The Widespread Pain Index (WPI) quantifies the number of body regions reported as painful by the patient. Other FMS symptoms like fatigue, poor sleep quality, cognitive, and somatic symptom were combined into a 0-12 symptom severity (SS) scale ${ }^{[12]}$. 
The current awareness of FMS has widened the perspective of the disease $\mathrm{e}^{[12]}$. Thus, the physicians are warranted to evaluate the patients with FMS regarding other aspects of health beyond the tender points and fatigue and the central symptoms of the disease $\mathrm{e}^{[13]}$. Because $\mathrm{SF}$ is strongly connected to physiological and psychosocial functioning, the physical and emotional symptoms of fibromyalgia (FM) could likely interfere with all dimensions of SF in affected patients ${ }^{[5,14]}$. Additionally, the medications used to manage FMS symptoms, such as antidepressants and mood stabilizers, have well-documented adverse effects on $\mathrm{SFs}^{[15,16]}$. We aimed to determine the rate of SD in drug-naive uncircumcised women with FM compared with pain-free matched controls and to appraise the potential predictors of sexual impairment in affected women.

\section{PATIENTS AND METHODS}

\section{Study design, setting, and participants}

This controlled, cross-sectional, single-center study was conducted in Andrology Department, Beni-Suef University Hospital in collaboration with Rheumatology Department, Faculty of Medicine, Beni-Suef University, from February 2017 to November 2018. A total of 50 consecutive women with FMS (patient group) and 50 pain-free women (control group) who met the inclusion criteria stated later and who showed interest to join the study were recruited. Eligible participants signed an informed consent after the study objectives and purpose were clarified to them according to the regulations of Review Ethical Committee of Beni-Suef Faculty of medicine that conforms to Helsinki Declaration (1964).

\section{Inclusion criteria}

The patients and controls were included only if they were in the reproductive age between 20 and 40 years, uncircumcised, married, and sexually active during the past month. Participants with female genital cutting were excluded, to preclude its negative effects on SF and to ascertain that the detected SD was forcefully related to the disease. Patients were drug naïve (i.e. never exposed to a definite therapy for FMS), diagnosed as having FMS, and recruited from the Rheumatology outpatient clinic according to the ACR classification criteria (2010) for $\mathrm{FMS}^{[12]}$. The $2010 \mathrm{ACR}_{\text {criteria }}{ }^{[12]}$ for diagnosis of FMS include the following: first, Widespread Pain Index (WPI) 7, Symptom Severity (SS) scale score 5 or WPI between 3 and 6, and SS scale score 9; second, symptoms have been present at a similar level for at least 3 months; and third, the patients do not have a disorder that would otherwise explain the pain.

Control group included otherwise healthy, agematched and education level-matched women who were companions to the female patients and reported no ongoing pain in any region of the body during the last 3 months.

\section{Exclusion criteria}

Women with gynecologic disorders such as polycystic ovary syndrome, inflammatory disorders such as rheumatoid arthritis, chronic disorders such as heart or liver disease, endocrine disorders such as diabetes or thyroid dysfunction, morbid obesity, major psychiatric or neurological disorders, lower urinary tract symptoms, antidepressant use, or a record of prescription medication or illicit drug abuse or alcoholism were excluded. Finally, women whose partners were had major systemic illnesses were also excluded from the study.

\section{Study procedures}

A demographic and clinical data collection form designed by the researchers was used to gather information about the characteristics of the study participants. Information about FMS in the form of onset, course, and duration of disease; pain sites; and severity was also gathered. The affected women were evaluated for tender point count (TPC), and pain level on a $0-10$-point visual analog scale (VAS). Additionally,FMS-related problems were estimated via Fibromyalgia Impact Questionnaire $(\mathrm{FIQ})^{[17,18]}$.

The validated Arabic version of the female sexual function index (ArFSFI) ${ }^{[19]}$ and the Arabic version of hospital anxiety and depression subscales (HADS-A and HADS-D ${ }^{[20,21]}$ were, respectively, used to assess SF and emotional symptoms of all participants.Moreover, the partners of the affected patients and controls were evaluated for SF by the validated Arabic version of the abridged five-item international index of erectile function $(\text { IIEF-5 })^{[22,23]}$. A structured interview was conducted to ensure confidentiality and privacy of the participants in which they were administered the questionnaires and asked to fill in the answers by themselves.

\section{Questionnaires}

The FSFI: the Arabic version of FSFI (ArFSFI) ${ }^{[19]}$ is a 19-item multidimensional scale. Specific domains analyzed in the ArFSFI are the quality of desire (questions 1 and 2), arousal (questions 3-6), lubrication (questions 7-10), orgasm (questions 11-13), satisfaction (questions 14-16), and degree of pain (questions 17-19). Each item is scored on a scale of 0 or 1-5. FSFI overall scores of less than 26.55 indicate impaired SF.

The HADS: the Arabic HADS ${ }^{[2,21]}$ includes anxiety and depression subscales. Each subscale consists of seven related items. Each item is rated from 0 to 3 . Scores of greater than or equal to 11 with either subscales indicate a definite case of psychiatric comorbidity, whereas scores of less than or equal to 7 with either subscales indicate a noncase of anxiety and depression, respectively.

The FIQ :the Arabic FIQ ${ }^{[17,18]}$ is a 10 -item, selfadministered questionnaire. The first item contains 11 
sub-items related to physical functioning. The answers are scaled in a Likert format from 0 to 3 . The scores on each sub-item are added together and divided by the number of valid answers to yield one physical impairment score. Items 2 and 3 ask the patient to mark the number of days they felt well and the number of days they were unable to work because of FM symptoms. Items 4 through 10 are horizontal linear scales marked in 10 increments on which the patient rates work difficulty, pain, fatigue, morning tiredness, stiffness, anxiety, and depression. Each of the 10 items has a maximum score of 10 . Thus, the maximum possible score is 100 .

The IIEF-5: the validated Arabic version of IIEF-5 ${ }^{[22,23]}$ is a five-item, self-administered questionnaire. Each item is scored on a scale of 0 or $1-5$. The possible scores for the IIEF-5 range from 1 to 25 , and a score above 21 is considered as normal erectile function and at or below this cutoff, erectile dysfunction (ED). ED is classified into four categories based on IIEF-5 scores: severe (1-7), moderate (8-11), mild to moderate (12-16), mild (17-21), and no ED ${ }^{[22-25]}$.

\section{Statistical methods}

Data were coded and entered using the Statistical Package for the Social Sciences version 25 (IBM Corp., Armonk, NY, USA). Data were summarized using mean and SD for quantitative data, whereas frequency and percentage were used for categorical data. Comparisons between quantitative variables were done using the nonparametric Mann-Whitney test. For comparing categorical data, $\chi^{2}$ test was performed. Exact test was used instead when the expected frequency is less than five. Correlations between quantitative variables were done using Pearson correlation coefficient. Potential predictors of SF in affected women were evaluated in a multivariate regression analysis to find out significant predictors. $P$ values less than 0.05 were considered as statistically significant.

\section{RESULTS}

The mean age of the affected women and controls was $30.42 \pm 4.99$ and $31.4 \pm 5.35$ years, respectively. The difference in age between cases and controls was not statistically significant $(P=0.345)$. Most women from both groups were housewives who had a pre-university education level. Both groups were comparable in terms of socio-demographic and clinical characteristics, anthropometric measures, as well as age of male partner $(P>0.05)$. However, patients with FM had lower frequency of sexual intercourse than control subjects $(1.70 \pm 0.79$ and $3.42 \pm 0.69$, respectively, $P<0.001$ ). In affected women, the mean duration of complaints related to FM was $2.42 \pm 1.72$ years; the TPC was $13.28 \pm 2.18$; the FIQ score was $47.50 \pm 21.34$ and the VAS was $4.86 \pm 2.19$ (Table 1).

Besides, the IIEF-5 scores of the partners of the controls were significantly higher than those of affected women (22.48 \pm 1.42 and 19.78 \pm 2.33 , respectively, $P<0.001)$. In addition, we encountered mild to moderate erectile dysfunction (ED) in the partners of affected women [19/50 (38\%) and 13/50 (26\%), respectively], whereas eight $(16 \%)$ partners of the healthy controls exhibited mild degree of ED. Our study revealed that the mean scores of both anxiety and depression subscales of HADS were significantly higher among patients $(11.6 \pm 4.68$ and $11.46 \pm 4.96$, respectively) when compared with controls (5 \pm 2.64 and $2.26 \pm 1.68$, respectively, $P<0.001$ ). (Table 1 ).

It was noted that $68 \%$ of women with FMS and $16 \%$ women in control group were regarded as having abnormal ArFSFI total scores $(P<0.001)$.(Table 1). Besides, FSFI overall and domain-specific scores were significantly lower among affected women than controls $(P<0.001)$ (Table 2$)$.

Table 1: Demographic and clinical characteristics of the study groups.

\begin{tabular}{lccc}
\hline & Cases & Controls & $P$ value \\
\hline Age (years) & $30.42 \pm 4.99$ & $31.4 \pm 5.35$ & 0.345 \\
Educational level & & & 0.488 \\
Secondary education & $39(78)$ & $36(72)$ & $14(28)$ \\
University & $11(22)$ & & 0.499 \\
Occupational status & & $15(30)$ & $35(70)$ \\
Employed & $12(24)$ & $4.60 \pm 1.23$ & 0.091 \\
Unemployed & $38(76)$ &
\end{tabular}




\begin{tabular}{|c|c|c|c|}
\hline Number of children & $2.45 \pm 1.91$ & $2.53 \pm 1.79$ & 0.075 \\
\hline BMI $(\mathrm{kg} / \mathrm{m} 2)$ & $25.62 \pm 2.74$ & $26.31 \pm 3.08$ & 0.098 \\
\hline Frequency of intercourse (per week) & $1.70 \pm 0.79$ & $3.42 \pm 0.69$ & $<0.001 *$ \\
\hline \multicolumn{4}{|l|}{ HADS } \\
\hline Anxiety subscale & $11.6 \pm 4.68$ & $5.00 \pm 2.64$ & $<0.001 *$ \\
\hline Depression subscale & $11.46 \pm 4.96$ & $2.26 \pm 1.68$ & \\
\hline \multicolumn{4}{|l|}{ FSFI total scores } \\
\hline Normal & $16(32)$ & $42(84)$ & $<0.001$ \\
\hline Abnormal & $34(68)$ & $8(16)$ & \\
\hline Age of male partner (years) & $37.48 \pm 4.14$ & $36.58 \pm 4.26$ & 0.078 \\
\hline \multicolumn{4}{|l|}{ IIEF-5 categories } \\
\hline Mild dysfunction & $19(38)$ & $8(16)$ & \\
\hline Moderate dysfunction & $13(26 \%)$ & 0 & $<0.001 *$ \\
\hline No dysfunction & $18(36)$ & $42(84)$ & \\
\hline IIEF-5 scores & $19.78 \pm 2.33$ & $22.48 \pm 1.42$ & $<0.001^{*}$ \\
\hline \multicolumn{4}{|l|}{ Disease related data } \\
\hline Duration (years) & $2.42 \pm 1.72$ & - & \\
\hline TPC & $13.28 \pm 2.18$ & - & \\
\hline VAS & $4.86 \pm 2.19$ & - & \\
\hline FIQ & $47.50 \pm 21.34$ & - & \\
\hline
\end{tabular}

Values are mean \pm SD or frequency (\%).

FIQ, Fibromyalgia Impact Questionnaire ; FSFI, Female Sexual Function Index ; HADS, Hospital Anxiety and Depression Scale ; IIEF-5, abridged five-item International Index of Erectile Function; TPC, Tender Point Count; VAS, Visual Analog Scale.

*Statistically significant. 
Table 2: FSFI (total and domain) scores of the participants

\begin{tabular}{|c|c|c|c|}
\hline & FM patients & Controls & $P$ value \\
\hline Desire & $3.23 \pm 1.51(1.20-6)$ & $5 \pm 1.16(2.40-6)$ & $<0.001 *$ \\
\hline Arousal & $3.31 \pm 1.60(1.20-6)$ & $4.86 \pm 1.23(1.80-6)$ & $<0.001 *$ \\
\hline Lubrication & $3.36 \pm 1.56(0.90-6)$ & $4.91 \pm 1.22(1.80-6)$ & $<0.001^{*}$ \\
\hline Orgasm & $3.46 \pm 1.66(1.20-6)$ & $5.03 \pm 1.23(2.40-6)$ & $<0.001 *$ \\
\hline Satisfaction & $3.46 \pm 1.79(1.20-6)$ & $4.99 \pm 1.29(1.60-6)$ & $<0.001 *$ \\
\hline Pain & $3.40 \pm 1.71(1.20-6)$ & $5.11 \pm 1.20(1.20-6)$ & $<0.001^{*}$ \\
\hline FSFI total scores & $20.21 \pm 9.42(6.90-36)$ & $29.87 \pm 6.94(11.50-36)$ & $<0.001 *$ \\
\hline
\end{tabular}

Values are mean $\pm \mathrm{SD}$ (minimum-maximum).

FM, Fibromyalgia; FSFI, Female Sexual Function Index.

*Statistically significant.

Moreover, non-significant correlations were revealed between the ArFSFI total scores and the following clinical parameters:female age $(\mathrm{r}=0.003 ; P=0.984)$, duration of marriage $(\mathrm{r}=0.025 ; P=0.866)$, number of children $(\mathrm{r}=-0.156 ; P=0.284)$, BMI $(\mathrm{r}=-0.007 ; P=0.960)$, as well as age of male partner $(\mathrm{r}=-0.054 ; P=0.708)$. On the contrary, frequency of intercourse per week presented highly significant positive correlations with total scores of the ArFSFI $(r=0.914, P<0.001)$. Additionally, the IIEF-5 scores of the affected women' partners showed highly significant positive correlations with total scores of the ArFSFI $(r=0.631, P<0.001)$. However, the duration of disease displayed highly significant negative correlations with total scores of the ArFSFI $(\mathrm{r}=-0.893, P<0.001)$ (Table 3).

Table 3: Correlations between clinical parameters and FSFI total scores in patients with fibromyalgia (n: 50).

\begin{tabular}{|c|c|c|c|c|c|c|c|c|}
\hline & $\begin{array}{c}\text { Age of } \\
\text { affected } \\
\text { female }\end{array}$ & $\begin{array}{c}\text { Duration of } \\
\text { marriage }\end{array}$ & $\begin{array}{c}\text { Number of } \\
\text { children }\end{array}$ & BMI & $\begin{array}{l}\text { Age of male } \\
\text { Partner }\end{array}$ & $\begin{array}{l}\text { Frequency of } \\
\text { intercourse }\end{array}$ & $\begin{array}{l}\text { Duration } \\
\text { of disease }\end{array}$ & IIEF-5 scores \\
\hline \multicolumn{9}{|c|}{ FSFI total scores } \\
\hline $\mathrm{r}$ & 0.003 & 0.025 & -0.156 & -0.007 & -0.054 & 0.914 & -0.893 & 0.631 \\
\hline$P$ & 0.984 & 0.866 & 0.284 & 0.960 & 0.708 & $<0.001^{*}$ & $<0.001^{*}$ & $<0.001^{*}$ \\
\hline
\end{tabular}

FSFI, Female Sexual Function Index; IIEF-5, abridged 5-item of the International Index of Erectile Function.

*Statistically significant. 
Table 4: Correlations between disease related data and FSFI total scores in patients with fibromyalgia $(n=50)$.

\begin{tabular}{|c|c|c|c|c|c|}
\hline & TPC & VAS & FIQ & HADS-A & HADS-D \\
\hline \multicolumn{6}{|c|}{ FSFI total scores } \\
\hline r & -0.942 & -0.954 & -0.953 & -0.947 & -0.929 \\
\hline$P$ & $<0.001$ & $<0.001$ & $<0.001$ & $<0.001$ & $<0.001$ \\
\hline
\end{tabular}

FIQ, Fibromyalgia Impact Questionnaire; FSFI, Female Sexual Function Index; HADS-A and HADS-D, Hospital Anxiety and Depression Subscales; TPC, Tender Points Count; VAS, Visual Analog Scale.

Table 5: Regression analysis: predictive factors for sexual function in women with (fibromyalgia).

\begin{tabular}{|c|c|c|c|c|c|c|c|}
\hline \multirow[b]{2}{*}{ Model } & \multicolumn{2}{|c|}{ Unstandardized coefficients } & \multicolumn{3}{|l|}{$\begin{array}{l}\text { Standardized } \\
\text { coefficients }\end{array}$} & \multicolumn{2}{|c|}{$95.0 \% \mathrm{CI}$ for $\mathrm{B}$} \\
\hline & $B$ & SE & $\beta$ & $t$ & $P$ value & Lower Bound & Upper Bound \\
\hline \multicolumn{8}{|l|}{ FSFI total scores } \\
\hline (Constant) & 32.924 & 2.794 & & 11.783 & $<0.001$ & 27.300 & 38.548 \\
\hline HADS-A score & $-0.786-$ & 0.165 & $-0.390-$ & $-4.776-$ & $<0.001$ & $-1.117-$ & $-0.455-$ \\
\hline VAS & $-1.707-$ & 0.331 & $-0.396-$ & $-5.151-$ & $<0.001$ & $-2.374-$ & $-1.040-$ \\
\hline Frequency of intercourse & 2.776 & 0.790 & 0.233 & 3.513 & 0.001 & 1.185 & 4.366 \\
\hline
\end{tabular}

CI, Confidence Interval; FSFI, Female Sexual Function Index; HADS-A, Hospital Anxiety Subscale; VAS, Visual Analog Scale.

\section{DISCUSSION}

The present study revealed no correlation between the ArFSFI overall scores and the age of affected women. Similar findings were reported by Yilmaz et al. ${ }^{[24]}$. On the contrary, Collado-Mateo et al. ${ }^{[25]}$ demonstrated higher prevalence of sexual problems among affected women aged more than 50 years.

We strictly tried to exclude cases with comorbidities such as inflammatory disorders or drug treatment to preclude their possible effects on SF,to confirm that SD was strongly related to FMS, as well as to avoid confounding the results. Thus, we excluded cases with female genital cutting, as it was found to be associated with reduced scores of the FSFI in all domain scores except the sexual pain domain ${ }^{[26,27]}$

Additionally, this study had demonstrated significant negative correlations between disease duration and total scores of the ArFSFI. Consistently, a recent meta-analysis conducted by Besiroglu and Dursun ${ }^{[13]}$ revealed a clear relationship between FMS and female sexuality for the global sexuality score and all the sub-items. In contrast, Yilmaz et al. ${ }^{[24]}$ revealed no correlation between the total FSFI score and the duration of complaints of affected patients $^{[24]}$. Furthermore, our study did not identify any correlation between total score of the ArFSFI and BMI, which could be seen in agreement withYilmaz et al. ${ }^{[24]}$.

Interestingly, the current study had shown highly significant correlations between the level of SD and pain severity on VAS scale. On the contrary, Yilmaz et al. ${ }^{[24]}$ did not find any correlation between total score of the ArFSFI and VAS scale, although their affected patients reported higher pain severity on VAS scale. Moreover, our study had demonstrated a strong correlation between total score of the ArFSFI score and TPC. In the same context, Burri et al. ${ }^{[16]}$ showed that patients with chronic widespread pain report more sexual pain and sexual distress compared 
with controls. Moreover, our study had revealed that affected patients had severe impairment of SF compared with age-matched healthy controls. This SD of affected women appeared to affect all domains, which was in agreement with previous published data ${ }^{[28]}$. Interestingly, our study reported that $68 \%$ of affected females had $\mathrm{SD}$, which could be seen lower than that reported by Rico-Villademoros et al. ${ }^{[14]}$ and Collado-Mateo et al. ${ }^{[25]}$. Admittedly, there is conflict in literature regarding the most affected aspect of SF in affected women, where Yilmaz et al. ${ }^{[24]}$ reported impairment in all FSFI domains among affected women compared with healthy controls, which could be seen similar to our results. Moreover, Shaver et $a l^{[6]}$ reported that affected women showed significant impairment of all aspects of SF in women with FMS except sexual desire. On the contrary, a study conducted by Prins et al. ${ }^{[5]}$ reported that affected patients had more problems with sexual desire and satisfaction. They hypothesized that only the psychological aspect was adversely affected in $\mathrm{FMS}^{[5]}$. Moreover, Burri et al. ${ }^{[16]}$ showed that women with chronic widespread pain reported more difficulties with lubrication, more sexual pain, and higher levels of sexual distress.

Our study had revealed that $16 \%$ of the controls reported abnormal SF that could be seen almost equivalent to that reported by Collado-Mateo et al..$^{[25]}$ and lower than that reported by Rico-Villademoros et al. ${ }^{[14]}$ Furthermore, a strong correlation was demonstrated between total score of the ArFSFI score and the FIQ score, which was in agreement with the studies of Yilmaz et al. ${ }^{[24]}$ and Tutuoglu et al. ${ }^{[29]}$.

Regarding our results, a higher rate of ED was noted in the partners of affected women compared with those of healthy women $(P<0.001)$, which could be seen in agreement with Tutuoglu et al. ${ }^{[29]}$. Finally, our study had shown that anxiety, pain severity, and frequency of intercourse were the only factors that remained strongly correlated with total scores of the ArFSFI after adjustment of the other factors. Consistently, Collado-Mateo et al. ${ }^{[25]}$ revealed that depression, anxiety, and tenderness were the most relevant symptoms associated with SD in affected women. On the contrary, Kool et al. ${ }^{[16]}$ and Burri et al. ${ }^{[30]}$ stated that low relationship satisfaction and high partner involvement might predict bad SF of women with FMS.

Possible limitations to the present study need to be identified. Although we used validated instruments for evaluating sexual problems, variation in SF between groups was based completely on the domain-specific FSFI scores without considering sexuality-related distress. This does not replace a clinical diagnosis of FSD. Furthermore, partners of women with FMS were not investigated for psychological variables and quality of life, which can be seen as one of the limitations of the present study. At the same time, including women with partners suffering of weak erection could interrupt the true evaluation of female SF. However, to avoid confounding the results, a multivariate regression analysis has been conducted and it was found that anxiety, pain severity, and frequency of intercourse were the only factors that remained strongly correlated with total scores of the FSFI after adjustment of the other factors. Finally, we were not able to use other evaluating tools to assess female SF as the conservative nature of our community limited us from discussing sexual-related issues openly even if a structured interview was conducted.

\section{CONCLUSION}

Our study demonstrated that FMS could disturb all dimensions of SF in women. The relatively high frequency of sexual impairment among affected women (68\%) was independently related to anxiety, pain severity, and frequency of intercourse, and consequently, managing sexual problems in women with FMS may necessitate a multidisciplinary approach with both pharmacological and non-pharmacological interventions. As $64 \%$ of the male partners of patients reported ED, further studies are needed to evaluate all dimensions of sexuality in partners of affected women and their association with poor SF of their spouses.

\section{COFLICT OF INTEREST}

There are no conflicts of interest.

\section{REFERENCES}

1. Woodard T L, Diamond M P. Physiologic measures of sexual function in women: a review. Fertil Steril2009; 92:19-34.

2. McCool M E, Theurich M A, Apfelbacher C. Prevalence and predictors of female sexual dysfunction: a protocol for a systematic review. Syst Rev2014; 3:1-5.

3. First M B, Wakefield J C. Diagnostic criteria as dysfunction indicators: bridging the chasm between the definition of mental disorder and diagnostic criteria for specific disorders. Can J Psychiatry 2013; 58:663-669.

4. Mease P. Fibromyalgia syndrome: review of clinical presentation, pathogenesis, outcome measures, and treatment. J Rheumatol2005; 32:6-21.

5. Prins M A, Woertman L, Kool M B, Geenen R. Sexual functioning of women with fibromyalgia. Clin Exp Rheumatol 2006; 24:555-561.

6. Shaver J L, Wilbur J, Robinson F P, Wang $\mathrm{E}$, Buntin M S. Women's health issues with fibromyalgia syndrome. J Womens Health (Larchmt) 2006; 15:1035-1045. 
7. Ryan S, Hill J, Thwaites C, Dawes P. Assessing the effect of fibromyalgia on patients' sexual activity. Nurs Stand2008; 23:35-41.

8. Gun K E, Uludag M U, Cidem M, Akman T O, Ozbayrak S, OrnekN I, et al. Sexual dysfunction in premenopausal women with fibromyalgia. Acta Med Mediterranea 2013; 1:429-3.

9. Castro I, Barrantes F, Tuna M, Cabrera G, Garcia C, Recinos M, Espinoza L R, et al. Prevalence of abuse in fibromyalgia and other rheumatic disorders at a specialized clinic in rheumatic diseases in Guatemala city. J Clin Rheumatol 2005; 11:140-145.

10. Batmaz I, Sarıyıldız M A, Dilek B, İnanır A, Demircan Z, Hatipoğlu N, et al. Sexuality of men with fibromyalgia: what are the factors that cause sexual dysfunction?. Rheumatol Int2013; 33:1265-1270.

11. Wolfe F, Smythe H A, Yunus M B, Bennett R $\mathrm{M}$, Bombardier C, Goldenberg D L, et al. The American College of Rheumatology 1990 criteria for the classification of fibromyalgia. Report of the Multicenter Criteria Committee. Arthritis Rheum1990; 33:160-172.

12. Wolfe F, Clauw D J, Fitzcharles M A, Goldenberg D L, Katz R S, Mease P, et al. The American College of Rheumatology preliminary diagnostic criteria for fibromyalgia and measurement of symptom severity. Arthritis Care Res (Hoboken)2010; 62:600-610.

13. Besiroglu M D H, Dursun M D M. The association between fibromyalgia and female sexual dysfunction: a systematic review and metaanalysis of observational studies. Int $\mathrm{J}$ Impot Res2019; 31:288-297.

14. Rico-Villademoros F, Calandre E P, RodríguezLópez C M, García-Carrillo J, Ballesteros J, HidalgoTallón J, García-Leiva J M. Sexual functioning in women and men with fibromyalgia. J Sex Med2012; 9:542-549.

15. Lewis R W, Fugl-Meyer K S, Corona G, Hayes R D, Laumann E O, Moreira E D Jr, et al. Definitions/ epidemiology/risk factors for sexual dysfunction. J Sex Med2010; 7:1598-1607.

16. Burri A, Hysi P, Clop A, Rahman Q, Spector T D. A genome-wide association study of female sexual dysfunction. PLoS ONE2012; 7:e350414.

17. Bennett R M, Burckhardt C S, Clark S R. The Fibromyalgia Impact Questionnaire: development and validation. J Rheumatol18:728-733.

18. El Naby M A, Hefny M A, Fahim A E, Awadalla $M$ A. Validation of an adapted Arabic version of fibromyalgia syndrome impact questionnaire. Rheumatol Int2013; 33:2561-2567.

19. Anis T H, Gheit S A, Saied H S, Al Kherbash S A. Arabic translation of female sexual function index and validation in an Egyptian population. J Sex Med2011; 8:3370-3378.

20. Zigmond A S, Snaith R P. The hospital anxiety and depression scale. Acta Psychiatr Sc and 1983; 67:361-370.

21. El Raufaie O E, Absood G. Validity study of the hospital anxiety and depression scale among a group of Saudi patients. Br J Psychiatry1987; 151:687-688.

22. Rosen R C, Cappelleri J C, Smith M D, Lipsky J, Peöa B M. Development and evaluation of an abridged, 5 -item version of the International Index of Erectile Function (IIEF-5) as a diagnostic tool for erectile dysfunction. Int J Impot Res1999; 11:319-326.

23. Shamloul R, Ghanem H, Abou Zeid A. Validity of the Arabic version of the sexual health inventory for men among Egyptians. Int $J$ Impotence Res2004; 16:452-455.

24. Yilmaz H, Yilmaz S D, Polat H A, Salli A, Erkin $\mathrm{G}$, Ugurlu H. The effects of fibromyalgia syndrome on female sexuality: a controlled study. J Sex Med2012; 9:779-785.

25. Collado-Mateo D, Olivares P R, Adsuar J C, Gusi N. Impact of fibromyalgia on sexual function in women. J Back Musculoskeletal Rehabil2020; 33:355-361.

26. Obermeyer C. The consequences of female circumcision for health and sexuality: an update on the evidence. Cult Health Sex2005; 7:443-461.

27. BergR, DenisonE, FretheimA. Psychological, social, and sexual consequences of female genital mutilation/ cutting: a systematic review of quantitative studies. Norwegian Knowledge Centre for the Health Services2010.

28. Tikiz C, Muezzinoglu T, Pirildar T, Taskn Eo, Frat A, Tuzun C. Sexual dysfunction in female subjects with fibromyalgia. J Urol2006; 174:620-623.

29. TutuogluA, BoyaciA, KocaI, CelenE, KorkmazN. Quality of life, depression, and sexual dysfunction in spouses of female patients with fibromyalgia. Rheumatol Int2014; 34:1079-1084.

30. Kool M B, Woertman L, Prins M A, Middendrop $\mathrm{H}$ V, Geenen R. Low relationship satisfaction and high partner involvement predict sexual problems of women with fibromyalgia. J Sex Marital Ther2006; 32:409-423. 